\title{
BRCA1/2 associated cancer susceptibility: a clinical overview
}

\author{
Georgios Lypas, M.D.* \\ 1st Department of Medical Oncology, Hygeia Hospital, \\ Erythrou Stavrou 4, 15123 Marousi, Athens, GREECE
}

Received 21 September 2015; Accepted 5 February 2016

\begin{abstract}
The most frequently identified genetic cause of breast cancer is the germline mutation of BRCA1 and 2 genes. The carriers of these mutations are at high risk for breast and ovarian cancers and increased risk for pancreatic and prostate cancers. Personal and family history are routinely used to identify individuals at risk for carrying such mutations. Genetic counselling is required to guide them through genetic testing and risk management. Positive testing corresponds to a deleterious mutation, whereas negative testing is considered as uninformative; variants of unknown clinical significance are also classified as uninformative.

The most effective risk reduction strategy involves bilateral prophylactic mastectomy and bilateral salpingo-0ophorectomy, eliminating $90 \%$ of breast and $97 \%$ of ovarian cancer risks, respectively. Before and until such surgery, surveillance with physical examination, mammography and magnetic resonance mammography, starting at 25-30 years of age, are recommended for the early diagnosis of breast cancer; semi-annual surveillance with physical examination, transvaginal ultrasound and serum CA-125 is recommended for women who have not had prophylactic surgery. Carriers diagnosed with breast cancer run a high risk for a new breast primary and this information should be used accordingly. PARP inhibitors is a new class of antineoplastic agents, already approved for advanced BRCA1/2 mutant (germline or somatic) ovarian cancer.

Multigene panel testing may follow a negative $B R C A$ genetic test, often identifying other genetic causes of cancer, expanding its role in oncology. Further adoption of PARP inhibitors, refinement in estimation of BRCA-associated cancer risks and wider population testing, through NGS technology, may become available in the near future.

Keywords: Brca1 • Brca2 • Cancer susceptibility • Cancer genetics • Ovarian cancer • Breast cancer • Genetic counseling • Hereditary cancer - Parp inhibitors • Prophylactic surgery • Surveillance

(c) De Gruyter Open
\end{abstract}

\section{Introduction}

A diagnosis of cancer is very often accompanied by several questions both from the patients and the physicians. "Why me?" is a question routinely phrased by most patients, reflecting not only a psychological distress but also a genuine search for an explanation on the causes of their disease. The largest percentage of them may not receive a concrete answer for this question. Still, for a small, but not a negligible portion, the cause will be concerned with an inherited cancer predisposition. In many, but not all families, this predisposition is clinically evident, as a number of cancer diagnoses in a family or an individual. However, as families are growing smaller in modern generations, inherited predisposition may be occult.
Genetic cancer predisposition varies in penetrance, i.e. the percentage of the individuals who will develop cancer, after inheriting a specific genetic alteration. On that basis, penetrance is classified as high, intermediate and low, conferring a relative increase in cancer risk of more than five to ten times, two to five times and less than two-fold respectively, in comparison to the average population's cancer risk [1].

More specifically, $5-10 \%$ of breast cancer and over $15 \%$ of ovarian cancer diagnoses are caused by heritable mutations in certain genes; BRCA1/2 are the most frequently identified mutated genes. Their role as cancer susceptibility genes has been established since 1993-1994, and there has been an exponential increase in knowledge about them since then [2-4]. 


\section{Molecular genetics of brca1/2 genes}

$B R C A 1$ \& 2 genes, located in chromosomes 17q21 and $13 q 12-13$ respectively, function normally as a DNA repair genes, through participation in the homologous recombination mechanism [2]. Functionally significant mutations result in defective homologous recombination repairing. Prevalence of such mutations is estimated at 1:400 for general population, though inter-population variations may exist. Ashkenazi Jews have a prevalence of $1: 40$, justifying perhaps a discussion for populationwide genetic screening.

$B R C A 1 / 2$ mutations are inherited in an autosomal dominant manner; de novo mutations are extremely rare, accounting for less than $1 \%$ of positive tests. Sanger sequencing is typically used to diagnose point mutations, whereas larger rearrangements (deletions/ duplications) are diagnosed with techniques such as MLPA. The spectrum of genetic variations for BRCA1 and 2 genes varies among populations, however a complete evaluation for both types of mutations, requires combination of both techniques for all populations. An exception probably concerns Ashkenazi Jewish families in which three specific mutations account for over $99 \%$ of all BRCA1/2 identified [3].

Studies performed in Greek population have described some interesting findings. More than $50 \%$ of all breast cancer patients with mutated BRCA1/2 seem to carry one of the six specific mutations [5]. Another study of 403 unselected triple negative breast cancer patients reported a prevalence of $16 \%$ mutations in the BRCA1 gene [6] much in keeping with international studies. Concerning ovarian cancer, a study including both the familial and non-familial ovarian cancer subjects identified a prevalence of $10 \%$ BRCA1/2 mutations in the overall study population [7].

\section{Penetrance/ cancer risks}

The penetrance of $B R C A 1 / 2$ genes is high. $B R C A 1$ is considered as more penetrant than BRCA2, as far as breast and ovarian cancers are concerned, while $B R C A 2$ may be more penetrant for pancreatic, prostate and male breast cancers. Estimates on specific cancer risks vary among cohorts and populations, and these risks cannot be distinguished in a clinically meaningful manner for specific populations. Table1 [8] summarises these risks.

There is an increasing evidence that subsequent generations of mutations carriers tend to develop
Table 1. BRCA $1 / 2$ associated cancer risks.

\begin{tabular}{|c|c|c|c|}
\hline \multirow{2}{*}{$\begin{array}{l}\text { Cancer } \\
\text { Type }\end{array}$} & \multirow{2}{*}{$\begin{array}{c}\text { General } \\
\text { Population Risk }\end{array}$} & \multicolumn{2}{|c|}{ Mutation Risk } \\
\hline & & BRCA1 & BRCA2 \\
\hline Breast & $12 \%$ & $50 \%-80 \%$ & $40 \%-70 \%$ \\
\hline $\begin{array}{c}\text { Second } \\
\text { primary breast }\end{array}$ & $\begin{array}{c}3.5 \% \text { within } 5 \text { years } \\
\text { Up to } 11 \%\end{array}$ & $\begin{array}{c}27 \% \text { within } \\
5 \text { yrs }\end{array}$ & $\begin{array}{c}12 \% \text { within } 5 \text { yrs } \\
40 \%-50 \% \text { at } \\
20 \text { yrs }\end{array}$ \\
\hline Ovarian & $1 \%-2 \%$ & $24 \%-40 \%$ & $11 \%-18 \%$ \\
\hline Male breast & $0.1 \%$ & $1 \%-2 \%$ & $5 \%-10 \%$ \\
\hline Prostate & $\begin{array}{c}15 \% \text { (N. European } \\
\text { origin) } \\
18 \% \text { (African } \\
\text { Americans) }\end{array}$ & $<30 \%$ & $<39 \%$ \\
\hline Pancreatic & $0.50 \%$ & $1 \%-3 \%$ & $2 \%-7 \%$ \\
\hline
\end{tabular}

malignancies at younger ages, supporting the existence of genetic anticipation in BRCA1/2 inheritance. Cancers tend to be diagnosed at ages 7-8 years younger in mutations - positive off-springs of pathogenic mutation carriers $[9,10]$.

Germline mutations in specific gene regions may correlate with certain phenotypes; there are reports associating increased ovarian cancer incidence with mutations in exon 11 (called ovarian cancer cluster region). But the relevant evidence is currently not strong enough to modify clinical management of individuals carrying these mutations [11].

\section{Genetic evaluation: counselling and testing}

Identification of mutation carriers is routinely based on clinical information, typically through a recent diagnosis of a BRCA-associated cancer in an individual (most frequently breast or ovarian) and of course, the availability of cancer family history. Histopathologic information may be crucial in this evaluation, since breast and ovarian cancers arising in this setting tend to have certain histopathological features. BRCA1associated breast cancers are most frequently $(80 \%)$ triple negative, whereas BRCA2 is associated with a sporadic phenotype, i.e. ER positive; interestingly HER2 positivity is rather rarely expressed in BRCA-related breast cancers, in contrast with Li-Fraumeni syndrome, where over $60 \%$ are HER2 positive [12]. Ovarian cancer is mostly high-grade serous in phenotype. 
Table 2. Elements of informed consent for genetic testing of cancer susceptibility [39].

1. Information on the specific genetic mutation(s) or genomic variant(s) being tested, including whether the range of risk associated with the variant will impact medical care

Implications of a positive and negative result

Possibility that the test will not be informative

Options for risk estimation without genetic or genomic testing

Risk of passing a genetic variant to children

Technical accuracy of the test including, where required by law, licensure of the testing laboratory

7. Fees involved in testing and counseling and, for DTC testing, whether the counsellor is employed by the testing company

Psychological implications of test results (benefits and risks)

9. Risks and protections against genetic discrimination by employers or insurers

10. Confidentiality issues, including, for DTC testing companies, policies related to privacy and data security

11. Possible use of DNA testing samples in future research

12. Options and limitations of medical surveillance and strategies for prevention after genetic or genomic testing

13. Importance of sharing genetic and genomic test results with at-risk relatives so that they may benefit from this information

14. Plans for follow-up after testing

The course from the suspicion of cancer susceptibility to the confirmation of a relevant germline mutation has to go through genetic counselling, which is the cornerstone of genetic evaluation and subsequent cancer risk management. It is usually provided by oncologists (or other cancer physicians) with special training or genetic counsellors. Consideration for genetic testing should lead to pre-test genetic counseling, and testing results should be also addressed during post-test counseling.

The questions and issues vary widely for individuals and families who receive genetic consultation, and they need to be addressed primarily. Age, prior diagnoses of cancer, family and marital status, priorities, values, and socio-economic issues are some of the different variables among individuals and families who seek genetic guidance. Most importantly, a person who will get tested is aware beforehand of the various outcomes, their meaning, their implications and their limitations, and so must be prepared for the next steps.

The recommended components of a typical cancer genetic counseling session, according to the US National Society of Genetic Counselors [13] include:

- $\quad$ Setting a mutual agenda for the session

- Addressing psychosocial issues and emotional concerns

- $\quad$ Taking a detailed medical and family history

- $\quad$ Providing risk assessment and risk counselling

- $\quad$ Directing an in-depth consent process for genetic testing, when applicable

- Disclosing results of genetic testing, when applicable
- Determining and communicating screening and management plans

- $\quad$ Summarising and planning for follow-up

A pre-test genetic counselling session structure, proposed at the Genetic Oncology Training Program of the Hellenic Society for Medical Oncology is as follows, with an average duration of 90 minutes.

1. Identifying the proband's motives, predominant questions, worries and priorities for genetic evaluation and testing

2. Reception of a detailed medical and family cancer history. That should be as extensive as possible. It usually covers five generations but should not be limited to less than three generations.

3. Mini cancer genetics course, explaining the nature of genes, cancer susceptibility inheritance

4. Understanding of cancer risk for mutation carriers

5. Pre-test probability estimation

6. Risk reduction and prevention options

7. Testing details and options

8. Discussion and preview of plans for all testing outcomes

9. Establishing next steps - follow-up.

At the end of a genetic counselling session, the counselee must be in position to provide his consent for genetic testing, after having received and processed adequate information for a number of issues (Table 2) [14]. This can be a challenging task for both parts of counselling, since the amount of information to be passed can be very large and difficult to process. Often it can become even more complicated by the psychosocial burden of a recent or a potential cancer diagnosis in the counselee's family or of a diagnosis for himself/herself.

Studies of the psychological impact of genetic testing have demonstrated a temporary increase of distress levels for individuals with deleterious mutations, which usually returns to the level before testing, while negative or inconclusive testing is associated with a progressive decrease in distress levels. Psychosocial assessment is part of the genetic counselling, and referral for specialist's support may be appropriate for some individuals.

Comparative studies have determined that genetic counselling for cancer predisposition through telephone contact may be as effective and psychologically reassuring as face-to-face counselling. This makes access easier for patients who would need to travel a long distance to receive their counselling. The only limitation with distance counselling would be physical findings, such as skin/mucosal lesions, indicative of rare syndromes (such as Cowden's, Peutz-Jeghers, etc.). 


\section{Genetic testing}

Genetic testing is offered to members of families, taking into consideration the probability of a positive result. As an attempt to quantify this probability, various models such as BRCAPRO, BOADICEA, Myriad Tables etc. may be utilised, using medical and family history as the input. Several limitations apply to these models, which seem to perform better in bigger families, with breast and ovarian cancer diagnoses, while they significantly vary in the type, amount of input and ease of use in general oncology practice (e.g. Myriad Tables) versus a more time and labour-consuming approach, more appropriate for a Hereditary Cancer Clinic (BRCAPRO, BOADICEA) [15]. Although there is no specific cut-off to dictate or deter testing, the estimated likelihood may facilitate communication with the person counselled. Empirically, a 5-10\% pre-test probability prompts the recommendation of genetic testing. Also, in some health insurance systems, use of these models is the basis to decide on the reimbursement of genetic testing.

A relatively simple and inclusive tool, which assists oncologists in their decision-making, is the use of National Comprehensive Cancer Network (NCCN) testing criteria, included in their widely accepted guidelines. These criteria involve all BRCA-associated malignancies, including prostate and pancreatic cancers and cover the possibility of limited family structure, a term used to describe an under-representation of females who have reached age 45 in a pedigree (Table 3 ). Individuals who fulfill the NCCN criteria should be referred for genetic counselling and offered genetic testing. Still, roughly one in four mutation carriers do not have a significant family or personal history [16].

Genetic testing is performed on germline DNA, usually extracted from peripheral blood leucocytes. Point mutations can be detected in Sanger sequencing, while large deletions/duplications and rearrangements are detected through methods such as MLPA. Point mutations are by far the most frequent type of mutations; however, large deletions/duplications and other rearrangements are routinely performed after a negative test.

Genetic testing in families without a known mutation can have three possible results: positive, corresponding to a deleterious mutation; negative, where no pathogenic mutation is diagnosed and variant of unknown clinical significance (VUS). VUS is a genetic variant, on which there is no conclusive evidence whether it has functional impact. VUS and negative BRCA1/2 testing results are classified as non-informative, meaning they cannot provide information explaining the phenotype that prompted the testing itself.

\section{Clinical implications for mutation carriers}

\section{Surveillance strategies}

Screening for breast cancer is of utmost importance in this category of women, who run a very high risk, and remains a central recommendation. Annual breast screening is mandated by all relevant guidelines, with conventional mammography and magnetic resonance mammography (MRM) [17]. MRM is routinely suggested, due to diagnostic limitations of conventional mammography in this population, for reasons such as breast density, rapid development of cancer lesions and higher frequency of atypical lesions (atypical hyperplasia, lobular in-situ neoplasia etc). Interval cancers are also frequent in BRCA1/2 carriers. Based on that observation, some physicians and patients prefer to alternate between their mammograms and MRM every six months. A study performed by a Chicago group evaluated semi-annual MRM screening versus the annual screening, and provided evidence for superior sensitivity in case of a more frequent screening. The strength of evidence has not been such to change the guidelines yet. The recommended age at first screening is 25 years, but may be even younger, if the youngest age of cancer in the family has been reported at age 30 or younger. Notably, in relevant studies, despite the higher detection rate, a benefit in survival with the adoption MRM has not been apparent [18, 19].

The risks of frequent screening, especially with MRM, include false-positive diagnoses, leading to unnecessary biopsies and/or surgeries, adding in financial and psychological costs, although there is evidence supporting a sharp reduction of false-positives after the first three to five MRMs [20,21]. Concerns have also been voiced on the impact of radiation administered during mammograms. An important flaw of this technique lies on the fact that supporting data come mainly from two studies, both retrospective, and with several limitations [22]. Therefore, conventional mammography retains its important role as a screening method.

There is no effective and routinely recommended screening for ovarian cancer. Studies of bi-annual use of CA-125 serum testing and transvaginal ultrasonography have only demonstrated mediocre outcomes: Seventy per cent of cancers diagnosed in an earlier study and $30 \%$ in a more recent one were already stage III or IV. Based on that, surgical prophylaxis is the riskreduction method of choice. Screening, as described, is recommended by NCCN guidelines for mutation carriers who want to retain their fertility or, for other reasons are unable or unwilling to undergo such surgery [16]. 
Table 3. Nccn testing criteria [16].

A. Individual from a family with a known deleterious BRCA1/BRCA2 mutation*•A

B. Personal history of breast cancer ${ }^{\circ}$ plus one or more of the following:

- Diagnosed age $\leq 45$ years

- Diagnosed age $\leq 50$ years with $\geq 1$ first-, second-, or third-degree blood relative (on the same side of the family) with breast and/or epithelial ovarian/ fallopian tube/primary peritoneal cancer at any age, or with a limited family history ${ }^{\Delta}$

- Two breast primaries ${ }^{\S}$ when first breast cancer diagnosis occurred $\leq 50$ years

- Diagnosed $\leq 60$ years with a triple negative breast cancer

- Diagnosed $\leq 50$ years with a limited family history ${ }^{4}$

- Diagnosed at any age with $\geq 1$ first-, second-, or third-degree blood relative (on the same side of the family) diagnosed with breast and/or epithelial ovarian/fallopian tube/primary peritoneal cancer $\leq 50$ years

- Diagnosed at any age with $\geq 2$ first-, second-, or third-degree blood relatives (on the same side of the family) with breast and/or epithelial ovarian/ fallopian tube/primary peritoneal cancer at any age

- Diagnosed at any age with $\geq 2$ first-, second-, or third-degree blood relatives (on the same side of the family) with pancreatic cancer or aggressive prostate cancer (Gleason score $\geq 7$ ) at any age

- First-, second-, or third-degree male blood relative (on the same side of the family) with breast cancer

- For an individual of ethnicity associated with higher mutation frequency (e.g. Ashkenazi Jewish), no additional family history may be required*

C. Personal history of epithelial ovarian/fallopian tube/primary peritoneal cancer

D. Personal history of male breast cancer

E. Personal history of pancreatic cancer or aggressive prostate cancer (Gleason score $\geq 7$ ) at any age with $\geq 2$ first-, second-, or third-degree blood relatives (on the same side of the family) with breast and/or ovarian cancer and/or pancreatic cancer or aggressive prostate cancer (Gleason score $\geq 7)$ at any age

F. Family history only

- First- or second-degree blood relative meeting any of the above criteria

- Third-degree blood relative with breast cancer ${ }^{\diamond}$ and/or ovarian/fallopian tube/primary peritoneal cancer with $\geq 2$ first-, second-, or third-degree blood relatives (on the same side of the family) with breast cancer (at least one breast cancer $\leq 50$ years) and/or ovarian/fallopian tube/primary peritoneal cancer

* One or more of these criteria is suggestive of hereditary breast/ovarian cancer syndrome that warrants further personalised risk assessment, genetic counselling, and often genetic testing and management. The maternal and paternal sides should be considered independently. Melanoma has been reported in some hereditary breast/ovarian cancer families.

- Patients who have received an allogeneic bone marrow transplant should not have molecular genetic testing via blood or buccal samples due to unreliable test results from contamination by donor DNA. If available, DNA should be extracted from a fibroblast culture. If the source of DNA is not possible, buccal samples can be considered, subject to the risk of donor DNA contamination.

$\Delta$ Individuals with a limited family history, such as fewer than two first- or second-degree female relatives or female relatives surviving beyond 45 years in either lineage, may have an underestimated probability of a familial mutation.

$\diamond$ For the purposes of these guidelines, invasive and ductal carcinoma in situ breast cancers should be included.

$\S$ Two breast primaries include bilateral (contralateral) disease or two or more clearly separate ipsilateral primary tumors either synchronously or asynchronously.

$¥$ Testing for Ashkenazi Jewish founder-specific mutation(s) should be performed first. Full sequencing may be considered if ancestry also includes nonAshkenazi Jewish relatives or other hereditary breast/ovarian cancer criteria are met. Founder mutations exist in other populations.

¥ Clinical judgement should be used to determine if the patient has reasonable likelihood of mutation, considering the unaffected patient's current age and the age of the female unaffected relatives who link the patient with the affected relatives. Testing of unaffected individuals should only be considered when an appropriate affected member is unavailable for testing. Significant limitations of interpreting test results for an unaffected individual should be discussed.

National Comprehensive Cancer Network, v3.2013

\section{Risk reduction interventions}

\section{Surgical}

Bilateral mastectomy is the risk reduction method with the highest impact in the reduction of breast cancer risk. Based on the rationale of removal of at-risk tissue, breast cancer risk drops by about $90 \%$. The remaining risk is due to remaining breast gland tissue. Therefore, in tissue-sparing techniques such as skin/nipple-areola sparing mastectomy, the risk may be higher than more radical ones [17].
Bilateral salpingo-oophorectomy is a very strong (perhaps the strongest, along with the breast screening) recommendation for all carriers of BRCA1/2 mutations who have completed their family history. The impact on ovarian cancer related mortality is significant and established; notably, when performed at premenopausal age, an additional benefit concerns the reduction of breast cancer risk by half for these women. All-cause mortality is reduced by $77 \%$ (HR $0.23,95 \% \mathrm{Cl} 0.13-$ $0.39, p<0.001)$ for these women $[16,23,24]$. These observations were recently reflected in a recent study, 
where preventive oophorectomy was associated with an $80 \%$ reduction in the risk of ovarian, fallopian tube or peritoneal cancers in BRCA1 or BRCA2 carriers and a $77 \%$ reduction in all-cause mortality, during a 5.6-year median follow-up [25].

Based on evidence of the fallopian tube as the site of origin for ovarian cancer, a trend for salpingectomy only is emerging. This evidence is not strong and the method is not a recommended alternative for bilateral salpingo-oophorectomy; moreover, the benefit of associated breast cancer risk reduction is missed with that intervention.

\section{Chemoprevention}

Chemoprevention for breast cancer in Europe has not been widely accepted, in contrast with the United States. SERMS, mainly Tamoxifen, are usually adopted for that indication. Evidence for the reduction of specific $B R C A 1 / 2$-associated breast cancer risk is very limited, and seems to be mostly about BRCA2 mutation carriers, though there is data for BRCA1 carriers as well. The explanation for this differential benefit is probably due to a predominance of ER-positive phenotype in BRCA2linked breast cancers, in contrast to the triple negativity of most BRCA1 breast cancers [26, 27].

Oral contraceptives have a well-established capacity of reducing ovarian cancer risk by at least $60 \%$. Their wide use for BRCA-caused ovarian cancer is limited by some evidence, suggesting a potential increase of breast cancer risk; this evidence comes from two studies, even though a large number of other studies do not confirm these findings. This controversy does not allow the routine use of oral contraceptives as a chemopreventive agent for ovarian cancer. More importantly, BRCA mutations carriers who have been taking oral contraceptives should still be advised to undergo preventive surgery [28, 29].

\section{Treatment considerations on carriers with cancer}

\section{Breast cancer}

There is no evidence of inferior local control in patients treated with breast-conserving therapy $(\mathrm{BCT})$ for breast cancer arising in patients with BRCA1/2 mutations, compared with sporadic cases. However, these patients run quite higher risk of a new primary, either at the ipsilateral or the contralateral breast (10-20\% vs $1-3 \%)$ ten years after the initial diagnosis. The lifetime risk of a new primary seems to be higher for women who are diagnosed young with their first breast cancer.
Among triple negative breast cancer tumours, BRCA1/2 mutation is a predictor of sensitivity in platinum-based agents, in comparison to taxanes. This differential effect was not observed BRCA1/2 wild-type triple negative breast cancers [30].

\section{Ovarian cancer}

Women diagnosed with BRCA1/2-associated ovarian cancer seem to have a better prognosis, including a better overall survival, probably due to an increased platinum sensitivity observed in this subpopulation.

Pharmacologic inhibition of the poly ADP ribose polymerase (PARP) is based on the concept of synthetic lethality, which exploits the homologous recombination deficiency (HRD) in BRCA-mutated tumour cells. The first of the class PARP inhibitor, olaparib, has been granted FDA and EMA approval for the treatment of advanced ovarian cancer positive for BRCA1/2 mutation. Importantly, this indication is not limited to germline mutation but also to somatic mutations, adding another $30-50 \%$ to the germline mutation carriers, corresponding to the number of patients carrying only somatic BRCA1/2 mutations [31].

\section{Negative and uninformative results: key role for post-test genetic counselling}

When a member of a family with a known BRCA1/2 mutation is tested for this mutation and is found negative, then it is considered as 'true negative', meaning that it does not carry the mutation considered responsible for this family's phenotype. True negative individuals are considered as at average population risk for breast and ovarian cancers. This information is of great value and significance for a relative of a deleterious mutation carrier, since her/his medical care and surveillance is that of average risk population and very importantly, it is news received with great relief.

In families without a known BRCA1/2 mutation, most tests will return with uninformative results, mostly negative and at times, VUS. These results mean that the individual's (and the family's phenotype) is not explained by a BRCA mutation and should be discussed during post-test genetic counseling. In that session, implications of this test are reviewed and further testing options are presented and discussed. Until a couple of years ago, genetic testing was concluded unless a phenotype indicative of another high penetrance gene (such as Cowden's, Li-Fraumeni, Peut-Jeghers, etc.) was considered worthwhile testing. This has been 
changing with the emerging and expanding availability of multiplex gene testing, through multi-gene panels.

It is now increasingly frequent for both counseling oncologist and counselees to pursue second-line testing for a group of genes related to breast and/or ovarian cancer. Such panels are now commercially widely available and they are considered as a cost-effective alternative to traditional single-gene testing strategy. Many of these panels include intermediate penetrance genes, whose clinical meaning and utility has severe limitation; for many genes, proper management is unclear, due to little amount of published evidence; other better studied genes, such as PALB2 or CHEK2, are accompanied by acceptable estimations on cancer risk for mutation carriers [32, 33]. Unfortunately it remains highly unclear whether true negative relatives run elevated risk and how severe it is. Due to that, counseling healthy, negative members of such a family remains highly problematic.

Another downside of panel testing has to do with unexpected results, where a deleterious mutations is detected in a gene that is irrelevant with the phenotype, which prompted the testing (e.g. $A P C$ in a family with breast and ovarian cancer diagnoses etc.). Though rare, the tested individual should be advised and warned on this possibility.

\section{Future directions}

It is almost certain that cancer risk in pathogenic $B R C A 1 / 2$ mutation carriers is subject to modifying factors. Recent data from a very large study (iCOGS) indicate the potential use of a common polymorphism panel, which can classify carriers in percentiles; top $5-10 \%$ of them are almost certain to develop breast or ovarian cancer, while the lowest 5-10 percentiles, may have as low as $50 \%$ lifetime risk for breast cancer and $10-15 \%$ for ovarian cancer [34, 35]. Validation of this panel could lead to additional testing for the refinement of risk estimates for carriers, leading to a more accurate and better justified clinical decisions and management. PARP inhibitors are, as abovementioned, a new class of anti-neoplastic agents. Promising studies are under way, investigating their role not only in breast and ovarian cancer, but also in other associated cancers such as pancreatic and prostate. What is more interesting is the testing not only of $B R C A 1 / 2$ in germline or tumour DNA, but its potential substitution from HRD assays. Such assays test the tumour for potential deficiency in its homologous recombination mechanisms, which may be subsequently exploited by PARP inhibition. So, it is possible in the near future that genomic testing may be the first step to identify tumour HRD, prompting subsequently a germline test with a panel including all relevant genes (BRCA1/2, ATM, BARD2, CHEK2, BRIP1, RAD51C,RAD51D etc), thus directing the testing flow from genomics to genetics.

Recently, M.C. King, a leading investigator of the identification of BRCA1 and 2 genes, at a provocative keynote lecture, while receiving the 2014 Lasker award, addressed the proposal for a population-wide BRCA testing [36]. Despite the significant controversies, risks and problems associated with such a public health strategy, an extensive discussion was kicked off due to that proposal. An important argument is the fact that approximately one-fourth of mutation carriers have no relevant family history, thus having no possibility of being warned that they are carriers and take appropriate protective measures. Population data supporting it come from Ashkenazi Jewish communities, where the prevalence of the mutation is very high (1:40). Moreover, three specific mutations are identifiable with a much less costly test than full gene sequencing and rearrangement testing. In contrast, most populations would require a much more expensive testing, with a much greater number of tests needed to identify the mutation carrier (average mutation prevalence is 1:400). That would require an explosive increase in clinical-genetics infrastructures to provide appropriate counselling. Even if compliance of deleterious mutation carriers in cancer surveillance and/or risk reduction interventions could be high enough to ensure cost-efficacy of the strategy, a large number would be found with VUS, making clinical management difficult. Besides, a colossal number of women would have uninformative (negative) results, leaving the question open on whether further testing would be required. A 2013 study of an oncology department in Greece identified the proportional of women fulfilling NCCN guidelines criteria, being over $40 \%$, while the percentage of the ones who were offered and underwent counselling and testing was miniscule [37]. A recently published study performed within a large US health insurance system, reported that among women who got BRCA genetic testing, the majority did not receive genetic counselling (61.8\%), with only $38.2 \%$ having been counselled by a genetics clinician. The primary reason for not getting counselling is the lack of referral by the physician who requested the testing. Women who did receive counselling reported a better level of understanding and satisfaction about their tests [38]. This result is rather surprising, contrasting relevant guidelines. It is the author's opinion that for many countries, effort and resources should be primarily allocated to the proper adoption of testing and management guidelines, until at least the proportion of 
women who get tested corresponds to the vast majority of those who fulfill testing criteria, and their further management is according to these guidelines.

\section{References}

[1] Stadler, Z.K., et al., Genome-wide association studies of cancer. J Clin Oncol, 2010. 28(27): p. 4255-67.

[2] Foulkes, W.D., Inherited susceptibility to common cancers. N Engl J Med, 2008. 359(20): p. 2143-53.

[3] Ford, D., et al., Genetic heterogeneity and penetrance analysis of the BRCA1 and BRCA2 genes in breast cancer families. The Breast Cancer Linkage Consortium. Am J Hum Genet, 1998. 62(3): p. 676-89.

[4] Yurgelun, M.B., E. Hiller, and J.E. Garber, Population-Wide Screening for Germline BRCA1 and BRCA2 Mutations: Too Much of a Good Thing? $\mathrm{J}$ Clin Oncol, 2015.

[5] Tsigginou, A., et al., Cumulative BRCA mutation analysis in the Greek population confirms that homogenous ethnic background facilitates genetic testing. Hered Cancer Clin Pract, 2015. 13(1): p. 17.

[6] Fostira, F., et al., Prevalence of BRCA1 mutations among 403 women with triple-negative breast cancer: implications for genetic screening selection criteria: a Hellenic Cooperative Oncology Group Study. Breast Cancer Res Treat, 2012. 134(1): p. 353-62.

[7] Stavropoulou, A.V., et al., Prevalence of BRCA1 mutations in familial and sporadic greek ovarian cancer cases. PLoS One, 2013. 8(3): p. e58182.

[8] Petrucelli, N., M.B. Daly, and G.L. Feldman, BRCA1 and BRCA2 Hereditary Breast and Ovarian Cancer, in GeneReviews(R), R.A. Pagon, et al., Editors. 1993: Seattle (WA).

[9] Litton, J.K., et al., Earlier age of onset of BRCA mutation-related cancers in subsequentgenerations. Cancer, 2012. 118(2): p. 321-5.

[10] Martinez-Delgado, B., et al., Genetic anticipation is associated with telomere shortening in hereditary breast cancer. PLoS Genet, 2011. 7(7): p. e1002182.

[11] Lubinski, J., et al., Cancer variation associated with the position of the mutation in the BRCA2 gene. Fam Cancer, 2004. 3(1): p. 1-10.

[12] Masciari, S., et al., Breast cancer phenotype in women with TP53 germline mutations: a LiFraumeni syndrome consortium effort. Breast Cancer Res Treat, 2012. 133(3): p. 1125-30.
[13] Riley, B.D., et al., Essential elements of genetic cancer risk assessment, counseling, and testing: updated recommendations of the National Society of Genetic Counselors. J Genet Couns, 2012. 21(2): p. 151-61.

[14] Robson, M.E., et al., American Society of Clinical Oncology Policy Statement Update: Genetic and Genomic Testing for Cancer Susceptibility. J Clin Oncol, 2015.

[15] Kurian, A.W., et al., Performance of prediction models for BRCA mutation carriage in three racial/ ethnic groups: findings from the Northern California Breast Cancer Family Registry. Cancer Epidemiol Biomarkers Prev, 2009. 18(4): p. 1084-91.

[16] NCCN, www.nccn.org (last accessed on $20 \mathrm{Jul}$ 2015).

[17] Daly, M.B., et al., Genetic/familial high-risk assessment: breast and ovarian. J Natl Compr Canc Netw, 2010. 8(5): p. 562-94.

[18] Saslow, D., et al., American Cancer Society guidelines for breast screening with $M R I$ as an adjunct to mammography. CA Cancer J Clin, 2007. 57(2): p. 75-89.

[19] Scheuer, L., et al., Outcome of preventive surgery and screening for breast and ovarian cancer in BRCA mutation carriers. J Clin Oncol, 2002. 20(5): p. $1260-8$.

[20] Bick, U., Intensified surveillance for early detection of breast cancer in high-risk patients. Breast Care (Basel), 2015. 10(1): p. 13-20.

[21] Chiarelli, A.M., et al., Effectiveness of screening with annual magnetic resonance imaging and mammography: results of the initial screen from the ontario high risk breast screening program. J Clin Oncol, 2014. 32(21): p. 2224-30.

[22] Narod, S.A., et al., Screening mammography and risk of breast cancer in BRCA1 and BRCA2 mutation carriers: a case-control study. Lancet Oncol, 2006. 7(5): p. 402-6.

[23] Kauff, N.D., et al., Risk-reducing salpingooophorectomy in women with a BRCA1 or BRCA2 mutation. N Engl J Med, 2002. 346(21): p. 160915.

[24] Rebbeck, T.R., et al., Prophylactic oophorectomy in carriers of BRCA1 or BRCA2 mutations. N Engl J Med, 2002. 346(21): p. 1616-22. 
[25] Finch, A.P., et al., Impact of oophorectomy on cancer incidence and mortality in women with a BRCA1 or BRCA2 mutation. J Clin Oncol, 2014. 32(15): p. 1547-53.

[26] Narod, S.A., et al., Tamoxifen and risk of contralateral breast cancer in BRCA1 and BRCA2 mutation carriers: a case-control study. Hereditary Breast Cancer Clinical Study Group. Lancet, 2000. 356(9245): p. 1876-81.

[27] King, M.C., et al., Tamoxifen and breast cancer incidence among women with inherited mutations in BRCA1 and BRCA2: National Surgical Adjuvant Breast and Bowel Project (NSABP-P1) Breast Cancer Prevention Trial. JAMA, 2001. 286(18): p. 2251-6.

[28] Cibula, D., et al., Oral contraceptives and risk of ovarian and breast cancers in BRCA mutation carriers: a meta-analysis. Expert Rev Anticancer Ther, 2011. 11(8): p. 1197-207.

[29] lodice, S., et al., Oral contraceptive use and breast or ovarian cancer risk in BRCA1/2 carriers: a metaanalysis. Eur J Cancer, 2010. 46(12): p. 2275-84.

[30] Tutt A, et al: The TNT trial. 2014 San Antonio Breast Cancer Symposium. Abstract S3-01. Presented December 11, 2014.

[31] Walsh, C.S., Two decades beyond BRCA1/2: Homologous recombination, hereditary cancer risk and a target for ovarian cancer therapy. Gynecol Oncol, 2015. 137(2): p. 343-50.

[32] Kurian, A.W. and J.M. Ford, Multigene Panel Testing in Oncology Practice: How Should We Respond? JAMA Oncol, 2015. 1(3): p. 277-8.
[33] Desmond, A., et al., Clinical Actionability of Multigene Panel Testing for Hereditary Breast and Ovarian Cancer Risk Assessment. JAMA Oncol, 2015. 1(7): p. 943-51.

[34] Gaudet, M.M., et al., Identification of a BRCA2specific modifier locus at $6 p 24$ related to breast cancer risk. PLoS Genet, 2013. 9(3): p. e1003173.

[35] Couch, F.J., et al., Genome-wide association study in BRCA1 mutation carriers identifies novel loci associated with breast and ovarian cancer risk. PLoS Genet, 2013. 9(3): p. e1003212.

[36] King, M.C., E. Levy-Lahad, and A. Lahad, Population-based screening for BRCA1 and BRCA2: 2014 Lasker Award. JAMA, 2014. 312(11): p. 1091-2.

[37] Georgios Lypas, K.P., Georgia Georgiou, Menelaos Zoulamoglou, Nikolaos Tsoukalas, Georgios Zografos, Pavlos Papakostas, Vasileios Barbounis, Identification of breast cancer patients fulfilling NCCN criteria for genetic risk assessment. J Clin Oncol 31, 2013 (suppl; abstr e12537).

[38] Armstrong, J., et al., Utilization and Outcomes of BRCA Genetic Testing and Counseling in a National Commercially Insured Population: The ABOUT Study. JAMA Oncol, 2015. 1(9): p. 1251-60.

[39] Robson, M.E., et al., American Society of Clinical Oncology policy statement update: genetic and genomic testing for cancer susceptibility. J Clin Oncol, 2010. 28(5): p. 893-901. 